\title{
25. Perioperative analgesia for the prolepsis of persistence of post-thoracotomy chronic neuropathic pain
}

Dionysia Boura $^{1}$, Eirini Anastassopoulou ${ }^{1,2, *}$, Paraskevi Koufopoulou ${ }^{3}$, Nikolaos Anastasiou ${ }^{4}$, Katsipoulakis Antonios $^{1}$, Lambropoulou Konstantina ${ }^{1}$

${ }^{1}$ Anesthesiology department, GONK "OI AG. ANARGYROI", Athens, Greece; ${ }^{2}$ Department of Nursing, School of Health \& Welfare Sciences, UNIWA, Athens, Greece; ${ }^{3}$ Department of Economics, Laboratory of Economics \& Health Management, School of Economics, Business \& International Studies, University of Piraeus, MSc. State \& Public Policy (Department of Management Science), Department of Political Science \& Public Administration, School of NOPE, EKPA, BSc. Of Social Policy \& Social Anthropology, Panteion University of Social \& Political Sciences, PPC SA, former Deputy Governor of GNA KAT, former Chairman of the Board KEKYKAMEA Cyclades, Athens, Greece; ${ }^{4}$ Thoracic surgery department, GONK “OI AG. ANARGYROI”, Athens, Greece. * ean1133@gmail.com

\begin{abstract}
Proper perioperative analgesic management of Thoracic surgery cases constitutes a primary factor for the successful outcome of the surgery, having created the ideal conditions for both the thoracic surgeon and the anesthesiologist, but mainly for the avoidance of postoperative pain for the patient. 5\%-67\% of thoracic surgery cases reports post thoracotomy pain syndrome that can persist for up to 20 years.

Purpose: To give prominence to the benefits of analgesics for the patient undergoing thoracic surgery after having received appropriate perioperative treatment and starts analgesic protection from the time preceding surgical incision to the patient's complete recovery, as far as prevention of chronic neuropathic pain is concerned. Complete analgesic protection presupposes the interdisciplinary collaboration of the surgeon and the anesthesiologist with the patient.

Materials \& methods: Thorough review of scientific literature in scientific databases (PubMed, Scopus, SignaVitae) concerning perioperative analgesia in thoracotomies.

Results: In our effort to prevent the persistence of Chronic Neuropathic Pain, we always take into consideration the fact that thoracotomy patients suffer from respiratory complications/uneasy breathing with invasive analgesia techniques. Therefore, we remain alert to reduce the use of opioids and their adverse effects on those patients. Major analgesic outcomes and avoidance of persistence of chronic neuropathic pain can be achieved with the following procedure: (1) Intravenous administration of non-steroidal anti-inflammatories, paracetamol, dexamethasone $8 \mathrm{mg}$, NMDA receptor antagonist (Ketamine $30 \mathrm{mg}$ ) before incision as well as local infusion of Ropivacaine $2 \%$ solution. (2) Intravenous infusion of lidocaine, fentanyl, remifentanil, morphine, tramadol, $\mathrm{Mg}$, ketamine, during surgery by adjusting doses per patient and operative time. (3) Particular caution is suggested in the immediate postoperative period so as to avoid transition from acute postoperative pain to chronic neuropathic pain. Our armamentarium includes paracetamol, tramadol, dexketoprofen, oxycodone per os, PCA morphine, pain busters (paired drainage catheters with elastomeric pumps) of ropivacaine $2 \%$, patch lidocaine and thoracic epidural analgesia with ropivacaine pumps and intercostal block nerve conduction. A medical record of the postoperative analgesic course of our patients is kept and completed by our medical and nursing staff.

Conclusions: Perioperative analgesia is our key concern in all thoracic surgeries, so as to prevent post thoracotomy pain syndrome. For the purpose of this concern, we administer analgesic treatment on all surgery stages as well as keep postoperative analgesic record.
\end{abstract}

